\title{
Relaxation Processes and Polymeric Chain Interpenetration in a PMMA-Cz/ MEH-PPV Blend Studied by Fluorescence Spectroscopy
}

\author{
T.D. Martins ${ }^{1, *}$ and T.D.Z. Atvars ${ }^{2}$ \\ ${ }^{1}$ Chemistry Institute, Federal University of Goias, P.O. Box 24242, Zip Cpde 74690-970, Goiania, GO, Brazil \\ ${ }^{2}$ Chemistry Institute, State University of Campinas, P.O. Box 6154, Zip Code 13084-971, Campinas, SP \\ Brazil
}

\begin{abstract}
Miscibility and energy transfer processes of a blend containing $0.1 \%$ of Poly[2-methoxy-5-(2-ethylhexyloxy)1,4-phenylenevinylene] (MEH-PPV) in a matrix of poly(methyl-methacrylate) containing $1 \%$ of attached carbazolyl groups (PMMA-Cz) were studied by fluorescence spectroscopy and microscopy techniques. From SEM micrographs, a uniform surface is found. The images on the cryogenic fracture of the blend does not show domains, although from fluorescence optical microscopy domains of MEH-PPV of about $30 \mu \mathrm{m}$ in the PMMA-Cz matrix were observed, indicating the immiscibility of the blend components. By steady-state and time-resolved fluorescence spectroscopies measured at room temperature and at $77 \mathrm{~K}$, it was found that there is an important non-radiative energy transfer occurring by Forster mechanism and due to that, there must be an interpenetration degree between polymeric chains specially at room temperature, which is evidence for miscibility. This can be inferred also by temperature-dependent fluorescence spectroscopy, which informs about relaxation processes occurring in the blend and also reveals the occurrence of an interface effect that is characteristic of miscible components.
\end{abstract}

Keywords: carbazole, FRET, MEH-PPV, chain interpenetration, PMMA, relaxation processes.

\section{INTRODUCTION}

New and more efficient luminescent materials with potential application in devices, solar cells and sensors have been suggested for the last two decades. Particularly those employing poly(phenylenevinylene) derivatives have attracted the attention of science and technology [1, 2]. Common strategies are to combine compounds with distinct photophysical properties in multi-layers, blends, doped films and membranes, in order to obtain the most efficient active layer for these devices, but aiming to distinct micromorphologies, leading to high ordered materials to minimize intragap electronic states related to defects [3], blends presenting long-lived exciton characteristics by limiting the recombination of polarons in their heterojunction [4], charge-transfer complexes formation that are taken as entities that can modulate the performance of organic solar cells by influencing the morphology of donor-acceptor blends [5] and even by influencing chain aggregation, which results in more efficient energy transfer processes due to effective interfacial interactions, at least when one of the compounds is in low content [6].

In the particular case of organic solar cells construction [7], most scientists have been focused on the approach of targeting the bilayer morphology with a

\footnotetext{
*Address correspondence to this author at the Chemistry Institute, Federal University of Goias, P.O. Box 24242, Zip Cpde 74690-970, Goiania, GO, Brazil; Tel: +55-62-3521-1097 (ext. 259); Fax: -55-62-3521-1093;

E-mail: tatiana@ufg.br
}

controlled thickness and consisting of a donor and an acceptor which permeates each other on a length scale of around $10 \%$ of the total thickness, in order to enable charge generation by the photoexcited electron-hole pair mechanism. The goal of this approach is to propose bilayers that are thin, capable of absorbing the total incident light and that would produce photoexcited eletctron-hole pair with great yield [8]. In sensor applications, the same goal is thought to be achieved, since thinner and more luminescent active layers are required to build the device, as deviations in the luminescence efficiency must evidence traces and a minimal quantity of a compound present in the sample. Thus, to an optoelectronic application, the need of a thin, multi-component active layer, with high efficiency in light absorption and charge transfer between components are the features that must be focused. Blends with isolating, low viscosity at higher temperatures polymers are thought to facilitate the components to permeate each other and the probability of charge transfer by resonant mechanisms would increase. Yet, to propose a blend for such uses, it is fundamental to carefully describe its photophysical and micromorphological behavior. In this work, the photophysics of a blend containing $0.1 \%$ in weight Poly[2-methoxy-5-(2-ethylhexyloxy)-1,4-phenylenevinylene] (MEH-PPV) in a poly(methyl methacrylate -comethacrylic acid)1,0:0,016 (Sigma-Aldrich Chemical Co. $\mathrm{Mn} \sim 15 \mathrm{~kg} \mathrm{~mol}-1$ e $\mathrm{Mw} \sim 34 \mathrm{~kg} \mathrm{~mol}^{-1}$ ) modified with 9-vinyl-carbazolyl fluorescent pendant groups (PMMA-Cz) matrix is detailed described by steady- 
state and time-resolved fluorescence spectroscopies. Its morphology and the degree of miscibility, interpreted in terms of chain interpenetration, between both components are studied and revealed by scanning electronic microscopy and fluorescence optical microscopy. Also, steady-state fluorescence spectra recorded in a range of temperature from 20 to $410 \mathrm{~K}$ revealed the relaxation processes that occur in the blend. Time-resolved fluorescence spectroscopic data obtained at two distinct temperatures also contributed to infer on charge transfer mechanisms and how they are affected by the system temperature.

\section{EXPERIMENTAL SECTION}

\section{Sample Preparation}

Blends were prepared from Poly[2-methoxy5-(2-ethylhexyloxy)-1,4-phenylenevinylene]-MEH-PPV (Sigma-Aldrich, $\mathrm{Mn}=84,000 \mathrm{~g} / \mathrm{mol}$ ) and a pre-obtained poly(methyl methacrylate -co- methacrylic acid) 1.0:0.016 ( $\mathrm{Mn} \sim 15 \mathrm{~kg}$ mol-1 e $\mathrm{Mw} \sim 34 \mathrm{~kg}$ mol-1) modified with 9-vinyl-carbazolyl fluorescent pendant groups- PMMA-Cz [9], was characterized by FT-IR spectroscopy, as showed elsewhere [9] and had its molar contents of the carbazolyl moieties determined spectroscopically. UV/Vis absorption spectra were recorded using a dual-beam Perkin-Elmer Lambda-6 spectrophotometer and a calibration curve from standard solutions of $9 \mathrm{HCz}$ in chloroform (with molar concentrations ranging from $5 \times 10^{-7}$ to $1 \times 10^{-4} \mathrm{~mol} \mathrm{~L}^{-1}$ ) was plotted. Molar absorptivity coefficient of carbazolyl units was determined at absorption wavelength of 332 $\mathrm{nm}$ as $0.401 \mathrm{~L} \mathrm{~mol}^{-1} \mathrm{~cm}^{-1}$. By using the experimental coefficient and registering the absorbance of solution of PMMA-Cz in chloroform, the molar amount of carbazolyl of $4.91 \times 10^{-5} \mathrm{~mol} \mathrm{~L}^{-1}$ was found.

A solution of $0.5 \mathrm{~g}$ of pre-obtained PMMA-Cz, as described elsewhere [9] dissolved in $10 \mathrm{~mL}$ of chloroform (Tedia, HPLC grade) and another of 0.0005 $\mathrm{g}$ of MEH-PPV dissolved in $2 \mathrm{~mL}$ of the same solvent, were mixed and with total solvent removal by casting technique, performed in a Petri dish kept under dynamic vacuum for $72 \mathrm{~h}$, a blend of PMMA-Cz/MEHPPV $0.1 \% \mathrm{~m} / \mathrm{m}$ is obtained. Resulting film thickness is $0.054(+/-0.003) \mathrm{mm}$, as measured by a MITUTOYO digital micrometer. Parts of this same film were employed in all further analysis described in the work.

\section{Methods}

Blend had its morphological characteristics and photophysical behavior studied by Differential Scanning
Calorimetry, Electronic Scanning and Fluorescence Optical Microscopies and by steady-state and time resolved fluorescence spectroscopic techniques.

Steady-state fluorescence spectroscopy was performed in an ISS-PCI spectrofluorimeter, operating with a $300 \mathrm{~W}$ Xe arc lamp and dynamic fluorescence measurements were performed in a time-correlated single photon counting (Edinburgh Analytical nF900 system) equipment, operating with hydrogen pulsed lamp, at a repetition rate of $40 \mathrm{kHz}$. The instrument response was determined using Ludox ${ }^{\circledR}$ as scatterer. Reconvolution of lamp pulse and sample signal was performed by non-linear least-squares routines, using software supplied by Edinburgh. Film samples were sealed under vacuum in quartz tubes and aligned at a $45^{\circ}$ angle to the incident radiation and the emission was collected at a right angle from the back face.

Temperature-dependent steady-state fluorescence spectra were recorded from 20 to $410 \mathrm{~K}$ under dynamic vacuum in a cryostat described elsewhere [10].

Electronic Micrographs were recorded in a JEOL JSM-6360-LV Electron Scanning Microscope (SEM), at an activation voltage of $20 \mathrm{kV}$. Sample surface was coated with thin layers of gold of about 100 angstroms. To perform fracture imaging, blend pieces were fractured under cryogenic conditions using liquid nitrogen.

Fluorescence optical emission micrographs were recorded in an inverted Leica DM IRB microscope employing a mercury arc lamp HBO (HBO-100 W) for UV-Vis excitation in the wavelength range of $330-380$ $\mathrm{nm}$, selected by optical filters. The emission image was selected from the excitation beam by a dichroic mirror $\left(\lambda_{\text {exc }}>410 \mathrm{~nm}\right)$. Objective magnifications of $25 \mathrm{X}, 50 \mathrm{X}$, and $100 \mathrm{X}$ were used, and the images were taken with a Samsung SDC-311 digital camera and processed by Linksys v. 2.38 software. Images show blue regions related to the fluorescence emission of the carbazoly units present in the PMMA-Cz matrix and the red emission of the MEH-PPV domains.

Differential Scanning Calorimetry (DSC) was performed in a Perkin Elmer DSC- 7 series, DuPont Instrument, model v2.2A 90, calibrated with Indium. Blend samples were analyzed at the range of temperature from 130 to $470 \mathrm{~K}$ and at the heating rate of $10 \mathrm{~K} / \mathrm{min}$. Cooling rate was $20 \mathrm{~K} / \mathrm{min}$ and two consecutive scans were performed. 


\section{RESULTS AND DISCUSSION}

\section{Photophysical Behavior of the PMMA-Cz/MEH-PPV Blend}

Steady-state fluorescence spectroscopy was performed at room temperature (approx. $298 \mathrm{~K}$ ) and at $77 \mathrm{~K}$. Excitation and fluorescence spectra of the blend sample was recorded and they are shown in Figure 1. Emission data was recorded in the wavelength range from 345 to $680 \mathrm{~nm}$ with excitation carried out at 340 $\mathrm{nm}$. From spectra in Figure 1, there is evidence for at least two distinct emission bands lying at distinct regions: a weak band at $350-400 \mathrm{~nm}$ exhibiting vibrational structure, with two well-distinguished peaks: a maximum at $348 \mathrm{~nm}$ and another at $365 \mathrm{~nm}$. Another intense band at $530-680 \mathrm{~nm}$ is observed, with maximum at $605 \mathrm{~nm}$ and a shoulder at $540 \mathrm{~nm}$ and another in $640 \mathrm{~nm}$. The excitation data was recorded in two ranges to avoid instrumental interference. The first range was recorded at a fluorescence wavelength of $352 \mathrm{~nm}$, the maximum of the first emission region and at the wavelength range from 260 to $350 \mathrm{~nm}$. The second was taken from 320 to $580 \mathrm{~nm}$, with emission wavelength at $600 \mathrm{~nm}$. Common region was used to create a baseline and keep the intensity ratio between the two excitation spectra.

To better explore the origins of the photopysical behavior of the blend, steady-state spectra were examined at split regions, one correspondent to data available for MEH-PPV common films and the other to data available on carbazole derivatives dilute solutions and of the PMMA-Cz, described in a previous work [9]. Both excitation and emission spectra are similar in shape and occurrence range to those obtained for the blend components separately. Also, since no emission from other emitting species is observed, it can be assumed as an evidence for blend composition.

Photophysical properties of both blend components were previously studied and some similarities between steady-state analysis of the blend and isolated components can be achieved. From earlier studies [1129] it was found that peaks at 348 and $365 \mathrm{~nm}$ are due to the emission of isolated carbazolyl units attached to the optically inert polymer matrix. At room temperature, emission from carbazole excimer is very weak and occurs at $440 \mathrm{~nm}$. Another study [30] showed that the band at 500-700 nm is characteristic of MEH-PPV. The peak at $605 \mathrm{~nm}$ is related to the emission of aggregated forms present in the polymeric film and the shoulder at $540 \mathrm{~nm}$ occurs due to the existence of nonaggregated polymer chains and it is not usually observed.

At $77 \mathrm{~K}$, band in the region of $340-400 \mathrm{~nm}$ is still weak. The peaks at $348 \mathrm{~nm}$ and $365 \mathrm{~nm}$ are shifted to longer wavelengths as the temperature is decreased. In the region of $450-700 \mathrm{~nm}$ there is a blue-shift effect on the peak at $605 \mathrm{~nm}$ more pronounced than the observed for the peak at $540 \mathrm{~nm}$, which show an intensity decrease as the temperature is increased more important than the former band. From 400 to 500 $\mathrm{nm}$ there is an emission band with vibrational structure similar to the phosphorescence already observed for the isolated PMMA-Cz at lower temperatures [9]. Nevertheless, as phosphorescence does not occur at room temperature, it may be related to the fluorescence emission of the partially overlapped excimer that is formed in the PMMA-Cz matrix and its phosphorescence. Excitation spectra were obtained at room temperature and at $77 \mathrm{~K}$. At both temperatures, the resulting spectra have the shape of a mixture of the
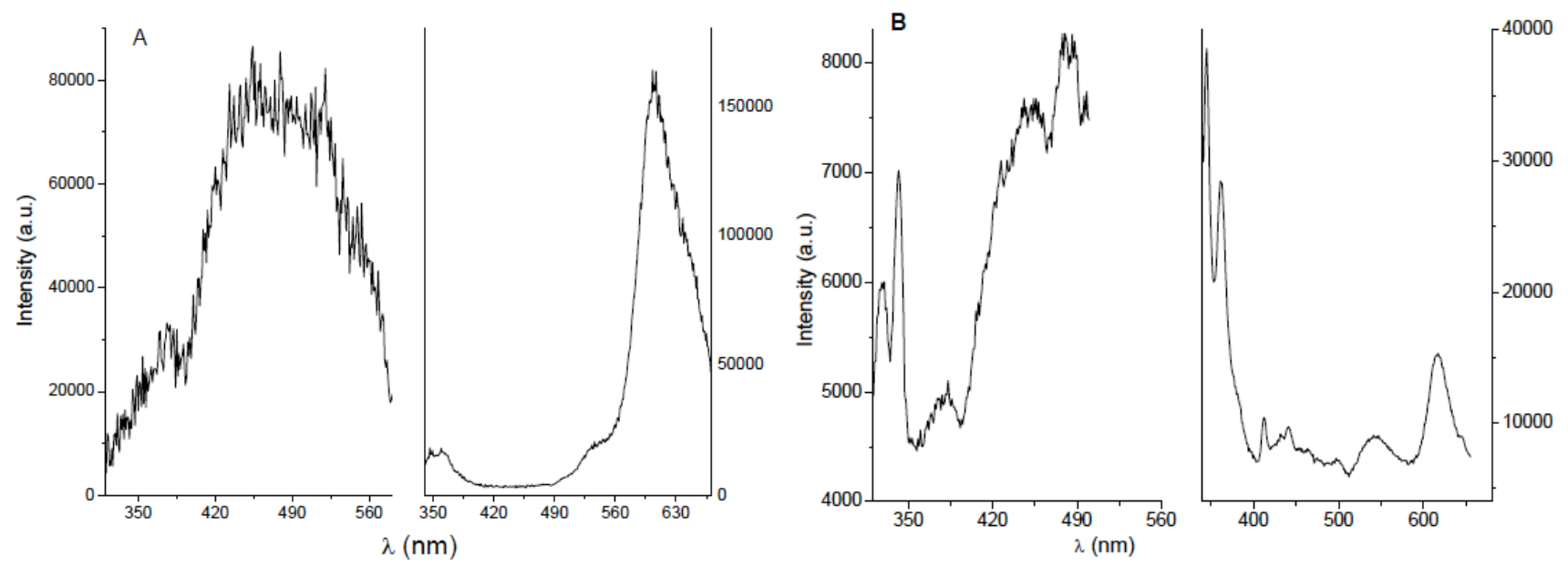

Figure 1: Excitation and fluorescence of PMMA-Cz/MEH-PPV blend at $\mathbf{A}$ ) room temperature and $\mathbf{B}$ ) at $77 \mathrm{~K}$. 
PMMA-Cz characteristic excitation spectrum and the MEH-PPV excitation spectrum, although at room temperature, PMMA-Cz portion is much less intense than that obtained at $77 \mathrm{~K}$. Emission and excitation spectra are shown in Figure 1. To better understand the effects of the temperature on the fluorescence intensity and shifts, emission spectra were recorded in a range of temperature from 20 to $410 \mathrm{~K}$.

\section{Temperature-Dependent Fluorescence Spectro- scopy}

Fluorescence spectra were recorded in two wavelength ranges, the first from 340 to $550 \mathrm{~nm}$, from where emission from carbazolyl-containing matrix is detected and from 430 to 700 nm, MEH-PPV domains' region of emission. Spectra are shown in Figure 2.

From the spectra taken at the latter wavelength range, related to MEH-PPV portion, the blue-shift and the intensity loss effects with increasing temperature are observed. This behavior is clearer in Figure 3, where peak positions (Figure $3 \mathbf{a}$ ) and the ratio between the peak intensities (Figure $\mathbf{3 b}$ ) are plotted against temperature. From these figures, blue-shifts of both MEH-PPV peaks are evident. Band peaked at $600 \mathrm{~nm}$ is almost $14 \mathrm{~nm}$ blue-shifted from its wavelength at the initial temperature, $20 \mathrm{~K}$, to its wavelength at the final temperature, $410 \mathrm{~K}$, while the band peaked at $540 \mathrm{~nm}$ is only $5 \mathrm{~nm}$ blue-shifted. From Figure $\mathbf{3 c}$, it is observed that the intensity ratio of the peaks at 540 and $600 \mathrm{~nm}$ decreases as the temperature increases, which shows that the intensity of the peak at $540 \mathrm{~nm}$ decreases faster than the intensity of the peak at 600 $\mathrm{nm}$, although it is not a linear relation. From 20 to 190 $\mathrm{K}$ it does not show an important decrease and the rate of intensity loss of both peaks is similar. From 190 to $320 \mathrm{~K}$ the decrease is intense, showing that at this temperature range, the intensity loss of the peak at 600 $\mathrm{nm}$ is slower than the intensity loss of the peak at 540 $\mathrm{nm}$. From 320 to $410 \mathrm{~K}$ it still decreases, although not drastically. Fluorescence intensity is expected to decrease as the temperature is raised, due to the increase in the rate constant of non-radiative processes that leads to vibronic deactivation, nevertheless, the
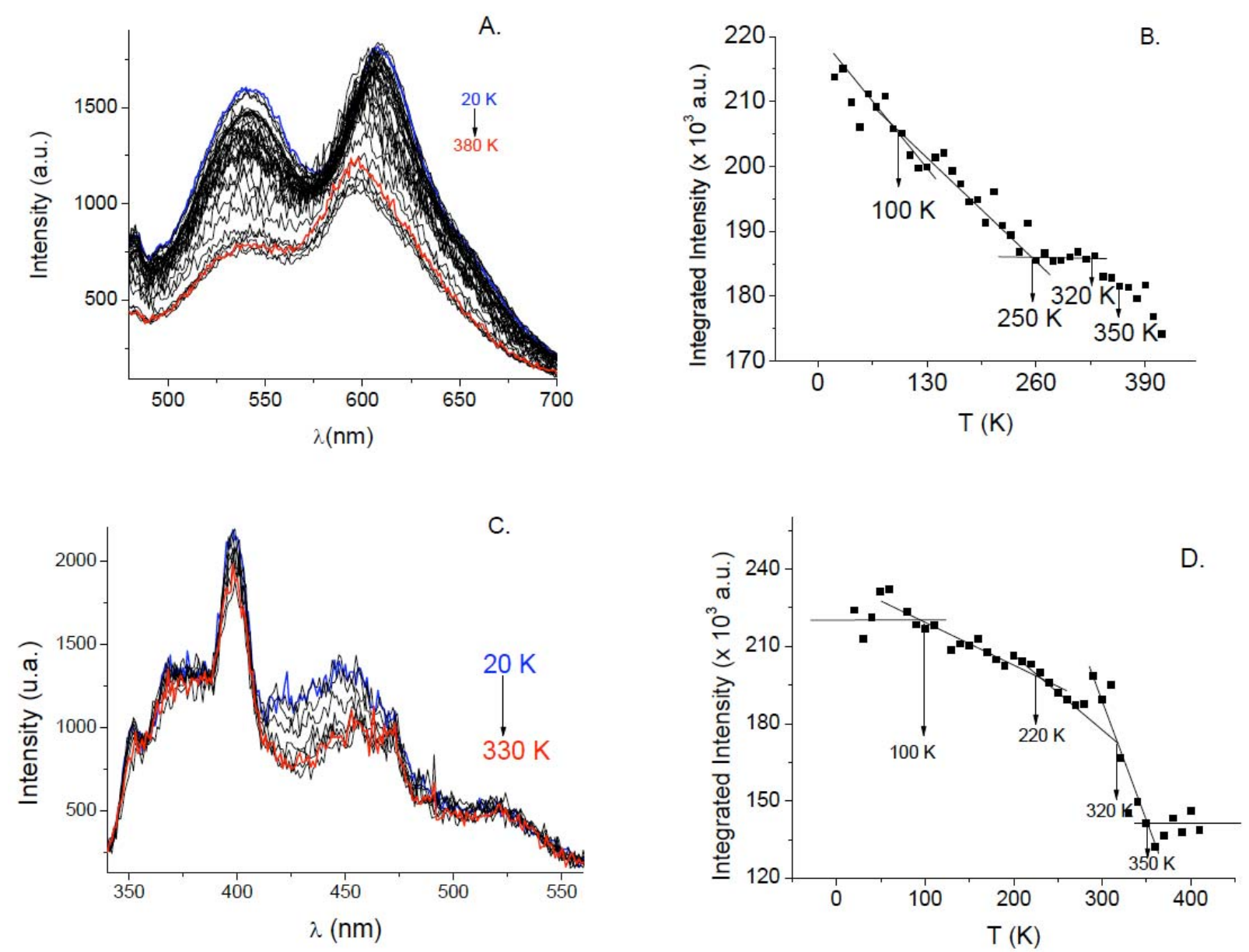

Figure 2: A) Fluorescence spectra and B) I $x \mathrm{~T}$ at the temperature range from 20 to $380 \mathrm{~K}$ and emission range of 450 to $700 \mathrm{~nm}$ $\left(\lambda_{\text {exc }}=430 \mathrm{~nm}\right)$ C) Fluorescence spectra $\left.\mathbf{D}\right)$ and I $x$ T at the temperature range from 20 to $330 \mathrm{~K}$. and emission range of 340 to $550 \mathrm{~nm}\left(\lambda_{\text {exc }}=330 \mathrm{~nm}\right)$. Some temperature data were omitted to permit behavior observation. 

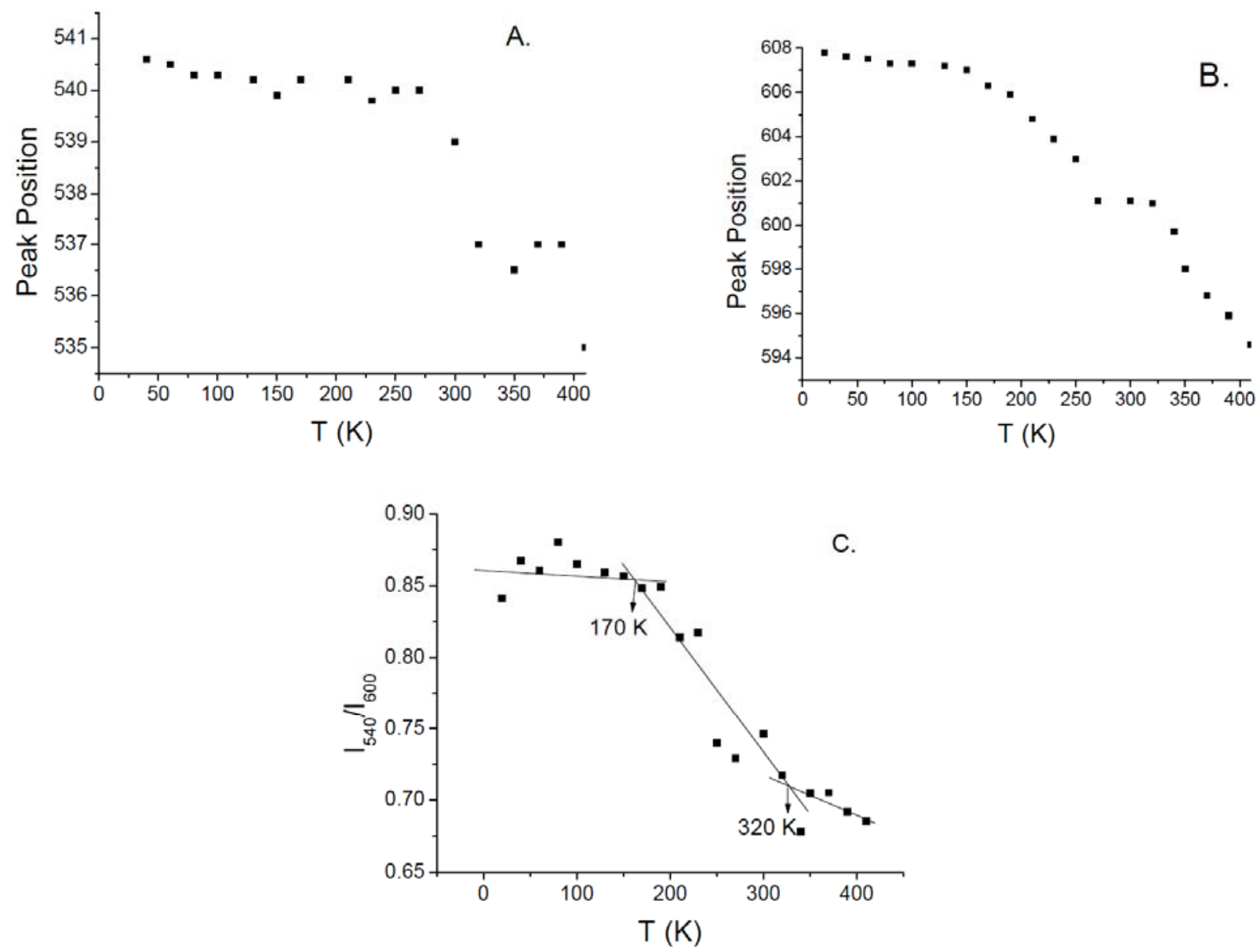

Figure 3: Graphic representation of peak position versus Temperature for A) band at $540 \mathrm{~nm}$ and B) $600 \mathrm{~nm}$ and C) intensity ratio between peaks at 540 and $600 \mathrm{~nm}$.

difference between deactivation rates of both peaks shows that the species that emits at $600 \mathrm{~nm}$, the interchain aggregated species, deactivates much slower than the species with emission at $540 \mathrm{~nm}$, the intrachain excitons [31]. Moreover, since the temperature-dependent fluorescence intensity is not a continuous function and as there is an isoemissive point at $576 \mathrm{~nm}$, which indicates interconversion between emissive species or equilibrium between them, the integrated intensity can be plotted against the system temperature, as an Arrhenius function. From this plot showed in the Figure $\mathbf{2} \mathbf{b}$ and $\mathbf{2} \mathbf{d}$, each change in the plot's angular coefficient represents a relaxation process undergoing in the components of the polymeric blend, at that particular temperature of inflexion. Relaxation processes can be identified at $100 \mathrm{~K}, 240 \mathrm{~K}$, $320 \mathrm{~K}$ and at $350 \mathrm{~K}$. As showed by Cossiello et al. [30], $\mathrm{MEH}-\mathrm{PPV}$ undergoes relaxation processes at $240 \mathrm{~K}$, related to the $\beta$ - relaxation and at $320 \mathrm{~K}$, related to the glass transition or $\alpha$ - relaxation.

Since there is no acceptable explanation for the changes at $100 \mathrm{~K}$ and $350 \mathrm{~K}$, they can be understood only in combination with data on the emission recorded at the wavelength range from where carbazolyl derivative is emitting. Arrhenius plot for this emission range was also constructed and is shown in Figure $\mathbf{2 d}$. Again, the same blend relaxation processes can be assigned by recording the fluorescence changes of the carbazolyl component and they are identified at $100 \mathrm{~K}$, $220 \mathrm{~K}, 320 \mathrm{~K}$ and $350 \mathrm{~K}$. These are the prominent processes, but not the only ones. For a trained eye, there are some unusual fluctuations in the data recorded, for example at 80 and $180 \mathrm{~K}$ (see Figure 2a and $\mathbf{2 c}$ ), that may be thought as minor processes occurring.

Relaxation processes in PMMA and PMAA matrices are well-known and have already been described [3242]. Changes in the fluorescence intensity of carbazolyl moieties that occurs at $100 \mathrm{~K}$ can be associated to motions of short macromolecular segments, methyl end-groups motions or rotation of the methyl groups bound to the main chain. These are designated as $\gamma$ relaxation. 
There is another process at $220 \mathrm{~K}$ that is attributed to motions of the PMMA's ester groups [35, 39-42]. The mechanism of these motions was described as a flip of the side groups [32]. The $\alpha$-relaxation process is observed at $350 \mathrm{~K}$. These facts corroborate with the relaxation process observed by DSC measurements, as shown in Figure 4. Virtually, the description of relaxation processes occurring in the blend as a whole can be achieved based on the recording of fluorescent response of any of its components, separately. Allied to that, there are some relaxation processes which are perceptible by accompanying the emission of each one of the blend components, for example those that might be occurring at $80 \mathrm{~K}$ and at $180 \mathrm{~K}$ (not marked in Figures $\mathbf{2} \mathbf{b}$ and $\mathbf{2 d}$ ), although they are not clear, and that cannot be assigned neither to the MEH-PPV nor to the PMMA portions by comparison with data already related to these components when pure. From that, it is evident that there is an interfacial effect acting in the fluorescence of each component, which is evidence of the existence of some degree of miscibility between the components of the blend. To explore this possibility of a degree of miscibility, microscopy techniques and by time-resolved fluorescence spectroscopy were performed.

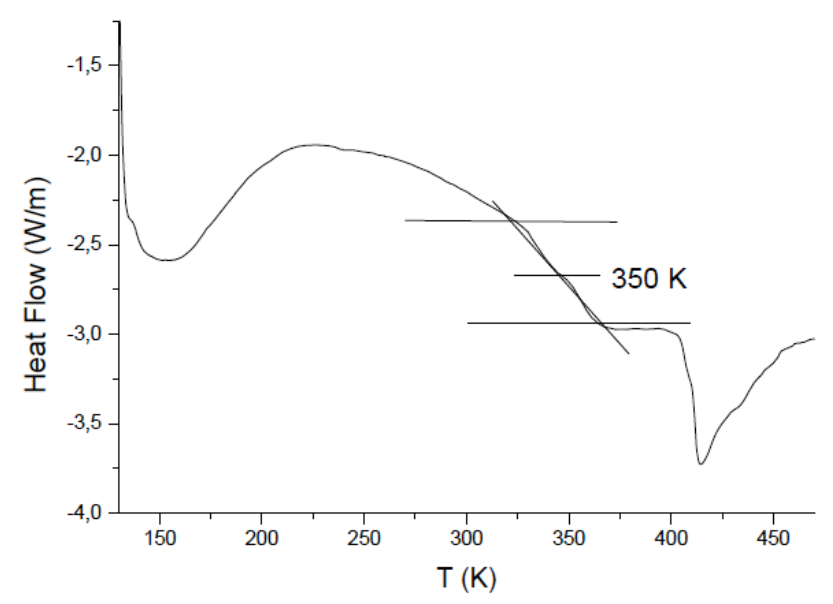

Figure 4: Differential Scanning Calorimetry traces recorded from the PMMA-Cz/MEH-PPV blend analysis.

\section{SEM and FOM Micrographs}

The blend's surface topology was analyzed by Scanning Electronic Microscopy (SEM) and Fluorescence Optical Microscopy (FOM) techniques, in order to observe its uniformity and, in addition, to identify MEH-PPV's domain sizes and distribution in the PMMA-Cz matrix. Micrographs are presented in Figure 5. By SEM technique uniform, domainless surface is observed (Figure $\mathbf{5 a}, \mathbf{b}$ ). Even when the facture is analyzed, no domains are observed (Figure 5c). Since most of the blend is constituted of the poly(methyl methacrylate) derivative, it does not support an electron beam too narrow and a sufficient zoom cannot be performed. From FOM measurements (Figure $5 \mathbf{d}, \mathbf{f}$ ), a blue-green matrix containing small red domains in disks form with diameter of up to $30 \mu \mathrm{m}$ are observed, which indicate immiscibility of the blend components. Similar morphology was obtained by lyengar et al. [43], where it was also found that the size of MEH-PPV depends on the composition of the blend. Nevertheless, when making use of the enhancing color image technique, some degree of miscibility can be inferred by the observation of some mixed colors regions, resulting in a green-yellow region around the orange-red domains and apart from the intense blue matrix (Figure 5e, g). Also, it is clear that there are color differences when images are taken at room temperature and at $77 \mathrm{~K}$. At room temperature, the blue color is important, whereas at $77 \mathrm{~K}$, the matrix assumes a green-blue color and a larger green-yellow colored area much extensive than at room temperature, at which the mixed colored area is limited the red MEH-PPV domains surroundings. These distinct color regions are indicative of a degree of miscibility susceptible to changes with temperature. Since the mixed colors are more important at lower temperatures, the blend components are miscible in a high degree and suffer a retraction back to their domains as the temperature increases and reptation motions are allowed to occur. Domain segregation is effective at around $240 \mathrm{~K}$, when beta-relaxation takes place $[35,39-42]$. To investigate this possible miscibility, time-resolved fluorescence was performed.

\section{Time-Resolved Fluorescence}

To infer about the miscibility degree of the components in the blend, fluorescence lifetimes were measured at room temperature and at $77 \mathrm{~K}$ and compared to the lifetimes obtained for each isolated component [17, 27, 44, 45]. Since this blend contains a small amount of MEH-PPV, relaxation processes cannot be rigorously identified by thermal analysis. Spectroscopic techniques are able to inform about morphology and miscibility in a more accurate way. Based on energy transfer processes between the components of the blend, one can infer about the blend miscibility. As the absorption spectrum of the MEHPPV domains are overlapped to the emission spectra of the carbazolyl unities attached to the PMMA matrix, proved by site-selective steady-state fluorescence spectroscopy, there is a possibility that this system undergo an energy transfer that can follow either a 


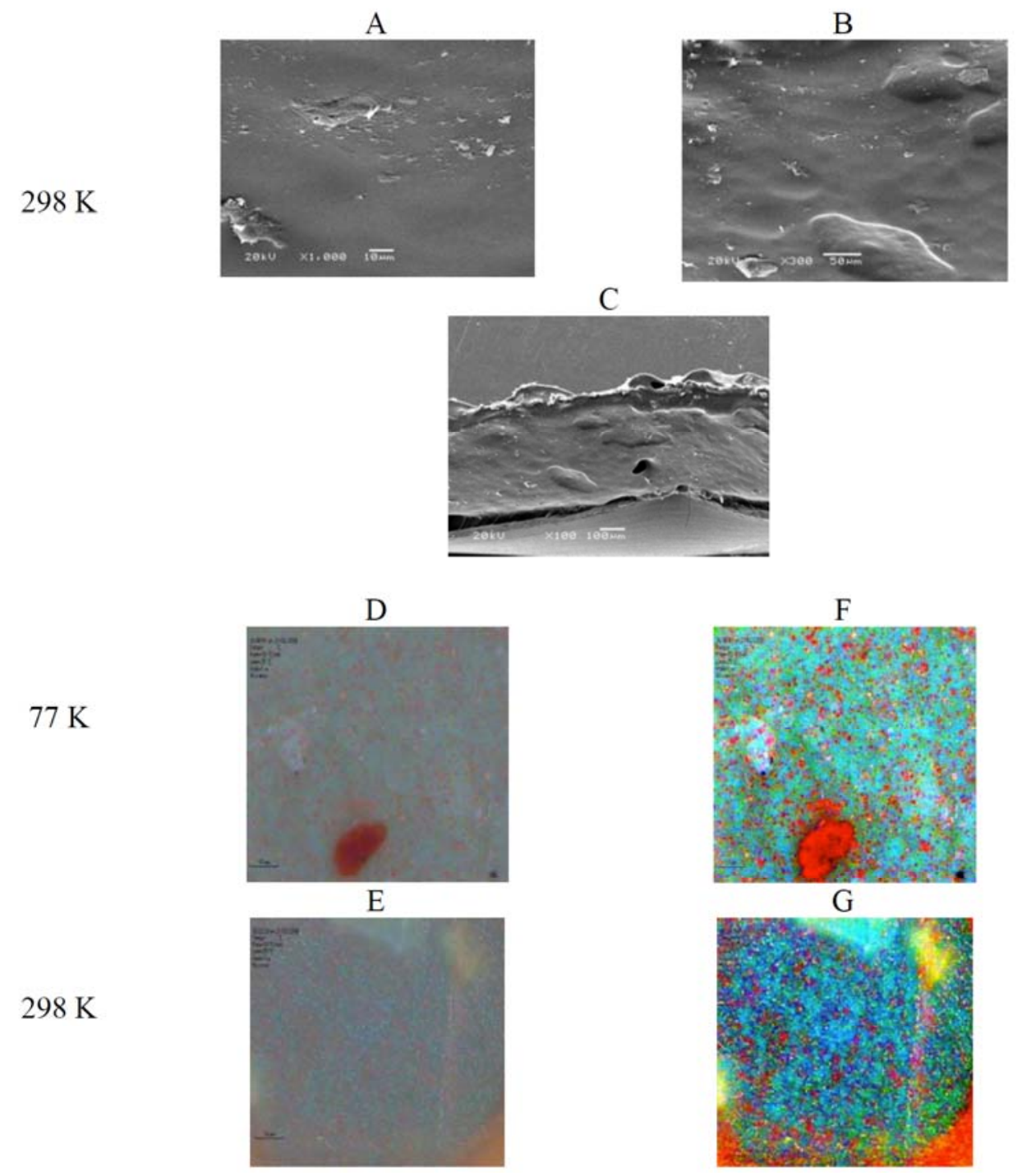

Figure 5: A, B) Surface scanning electronic (magnifications of X 200 and X 300); C) Fracture and D) Fluorescence optical micrograph of the PMMA-Cz/MEH-PPV blend (X 100) at $77 \mathrm{~K}$ and $\mathrm{E}$ ) at room temperature, with the respective enhanced color intensity images: F) FOM enhanced color image for the $77 \mathrm{~K}$ micrograph and G) FOM enhanced color image for the room temperature micrograph.

radiative or a non-radiative pathway. Since nonradiative energy transfer processes (NRET) are strongly dependent on the spatial arrangement of the participating components on the process, dynamic fluorescence spectroscopy techniques may be employed to discern upon both paths. From Forster theory [46], this range must be of a few nanometers for the energy transfer process to occur. Therefore, it is essential the interpenetration of the blend components at the molecular level, which means, in this case, a degree of miscibility. In Forster mechanism, NRET occurs when there are inductive forces acting on the components by means of dipole-dipole interactions, with efficiency $E_{T}$ that increases as the distance $r$ between donor and acceptor decreases. The rate $\left(k_{D A}\right)$ which this process occurs is given by:

$$
k_{D A}=\frac{9000 k^{2} \ln 10}{128 \pi^{5} n^{4} N_{A} \tau_{D A} r^{6}} \int \frac{F_{D}(\tilde{v}) \varepsilon_{A}(\tilde{v})}{\tilde{v}^{4}} d \tilde{v}
$$

Where:

$k^{2}$ is a factor that describes the relative orientation of the donor and acceptor dipoles; $N_{A}$ is the Avogadro's number; $F_{D}$ is the corrected donor fluorescence intensity normalized to the unit area; $\varepsilon_{A}$ acceptor molar extinction coefficient; $\tau_{D A}$ is the donor fluorescence lifetime in presence of the acceptor; $r$ is the donor-acceptor separation distance; $n$ is the refractive index. Since:

$$
k_{D A}=\frac{1}{\tau_{D}}\left(\frac{R_{0}}{r}\right)^{6}
$$


$\mathrm{R}_{0}$, Forster distance, is given by:

$$
R_{0}^{6}=\frac{9000 k^{2} Q_{D} \ln 10}{128 \pi^{5} n^{4} N_{A}} \int \frac{F_{D}(\tilde{v}) \varepsilon_{A}(\tilde{v})}{\tilde{v}^{4}} d \tilde{v}
$$

Where $Q_{D}$ is the donor fluorescence quantum yield.

According to these equations, the energy transfer process depends on the spectral overlap between the donor emission and the acceptor absorption. Since in the non-radiative process, the energy transfer must occur between the resonant excited states of a donor and an acceptor, it occurs in a time-range shorter than the donor's fluorescence lifetime. Therefore, NRET efficiency $\left(E_{T}\right)$ can be evaluated by the fluorescence lifetimes of the donor in presence and absence of the acceptor.

$$
E_{T}=1-\left(\frac{\tau_{D A}}{\tau_{D}}\right)
$$

Where $\tau_{D A}$ is the donor fluorescence lifetime in the acceptor's presence and $\tau_{D}$ is its lifetime in the acceptor's absence.

From these relations, decays will be monoexponential whenever donor and acceptor energy transfer occurs in a single distance. On the other hand, if there are several distances from which the energy transfer can occur, the decay curve must be described by a more complex exponential function and present a distribution of lifetimes. PMMA-Cz, in the absence of an acceptor, presents a bi-exponential decay curve at room temperature with two well-defined lifetimes, the longer one of $12 \mathrm{~ns}$ and the shorter one of 3-4 ns [9]. MEH-PPV, at room temperature, presents a shorter lifetime of 1-2 ns [31]. For the blend, fluorescence lifetimes were taken at five emission wavelengths characteristics of each component, i.e., at $350,367 \mathrm{~nm}$, from where only carbazolyl units emission can be detected, at $390 \mathrm{~nm}$, the emission region of a partially overlapped carbazolyl excimer, $430 \mathrm{~nm}$, emission region of the sandwich excimer and at $580 \mathrm{~nm}$, the emission region of the MEH-PPV component. For the first four wavelengths, shorter lifetimes in comparison to the values recorded for the isolated PMMA-Cz were obtained, although the fluorescence lifetime of $\mathrm{MEH}$ PPV is very similar to the obtained for the isolated polymer. Table 1 shows the fluorescence lifetimes for the system recorded at room temperature and at $77 \mathrm{~K}$. At room temperature, lifetimes that were expected to be near $12 \mathrm{~ns}$, were of $8 \mathrm{~ns}$ when recorded at $350 \mathrm{~nm}$ and, as the wavelength of monitoring is moved to the red, the lifetime is even shorter. At $430 \mathrm{~nm}$, the emission lifetime that was expected to be of $4 \mathrm{~ns}$, presented a value of 2 ns. The shortening of the lifetimes characterizes the energy transfer as occurring by the Forster mechanism, where the proximity of the PMMA-Cz donor to the acceptor MEH-PPV chains promotes the energy transfer from the carbazolyl and carbazolyl aggregates to the MEH-PPV moieties, in a non-radiative resonant mechanism. At $77 \mathrm{~K}$ the effect is also observed, but in a less expressive way. This effect might be due to two reasons: one is that since at low temperatures the system is as frozen and some chain motions are prohibited, it yields a less effective nonradiative energy transfer process. On the other hand, making use of the Forster equation to calculate the distance between the two luminescent components of the blend at both temperatures, shorter values of Forster radius is obtained at lower temperature. By employing the molar extinction coefficient of MEH-PPV as $3 \times 10^{4} \mathrm{~mol}^{-1} \mathrm{~cm}^{-1}$ [47] and the refractive index of the blend as the PMMA's, since MEH-PPV is present in such a proportion that might not alter the refractive index in an important matter, the Forster's overlap integral at room temperature results in a Forster radius of $6.05 \mathrm{~nm}$, as for $77 \mathrm{~K}$, with refractive index determined by Singh and Cariou methods [48] [49] and with a much higher phosphorescence quantum yield, a Forster radius of $5.62 \mathrm{~nm}$ is obtained, a much shorter radius than at room temperature, which evidences

\begin{tabular}{|c|c|c|c|c|c|c|}
\hline \multirow[t]{2}{*}{$\lambda_{\mathrm{em}}(\mathrm{nm})$} & \multicolumn{3}{|c|}{ Room Temperature } & \multicolumn{3}{|c|}{$77 \mathrm{~K}$} \\
\hline & $\tau_{1}(\mathrm{~ns})$ & $\tau_{2}(\mathrm{~ns})$ & $\chi^{2}$ & $\tau_{1}(\mathrm{~ns})$ & $\tau_{2}$ (ns) & $\chi^{2}$ \\
\hline 350 & $8.92(92 \%)$ & $1.67(9 \%)$ & 1.112 & $11.94(94 \%)$ & $1.90(6 \%)$ & 1.127 \\
\hline 367 & $8.83(89 \%)$ & $1.30(1,5 \%)$ & 1.120 & $10.69(86 \%)$ & $1.30(5 \%)$ & 1.241 \\
\hline 390 & $7.40(81 \%)$ & $1.05(19 \%)$ & 1.096 & 8.97 (79\%) & $1.10(21 \%)$ & 1.121 \\
\hline $430^{*}$ & $2.25(84 \%)$ & $0.24(14 \%)$ & 1.164 & $3.80(27 \%)$ & $0.20(21 \%)$ & 1.292 \\
\hline 580 & $1.44(33 \%)$ & $0.44(47 \%)$ & 1.106 & $2.50(39 \%)$ & $0.40(44 \%)$ & 1.015 \\
\hline
\end{tabular}

Table 1: Fluorescence Lifetimes Obtained at Room Temperature and $77 \mathrm{~K}$, at Several Emission Wavelengths

${ }^{*} \lambda_{\mathrm{exc}}=345 \mathrm{~nm}$. 
distinct interpenetration relations at both temperatures. Software PhotochemCad version 2 was used to corroborate these distances, by using the same refractive indexes mentioned before, the experimental data for fluorescence lifetimes of carbazolyl units and the fluorescence quantum yields at both temperatures. The resulting radii values were $5.86 \mathrm{~nm}$ at room temperature and $5.38 \mathrm{~nm}$ at $77 \mathrm{~K}$. Although values are not the same, they conserve the difference pattern and confirm that radius at $77 \mathrm{~K}$ is shorter than at room temperature. These calculations also gave the Forster energy transfer rates at room temperature and at $77 \mathrm{~K}$ and they were $1.81 \times 10^{25} \mathrm{~s}^{-1}$ and $6.36 \times 10^{24} \mathrm{~s}^{-1}$, respectively, which indicates that Forster mechanism is favored at higher temperatures.

MEH-PPV fluorescence lifetime is also slightly longer at lower temperatures and it is about 2 ns. The blend's longest fluorescence lifetime is obtained at 350 $\mathrm{nm}$ and it is well-fitted by a bi-exponential fit, which results in the lifetime values of $11 \mathrm{~ns}$ and $1.4 \mathrm{~ns}$ that are longer than the lifetimes obtained at room temperature, but still shorter than the values obtained for the isolated polymer. At longer wavelengths, at room temperature, the fluorescence lifetime is reduced to $10 \mathrm{~ns}$ at $370 \mathrm{~nm}$ and $9 \mathrm{~ns}$ at $390 \mathrm{~nm}$. At $430 \mathrm{~nm}$ it is $3.8 \mathrm{~ns}$ and is close to the excimer lifetime in the isolated PMMA-Cz, which is almost 4 ns.
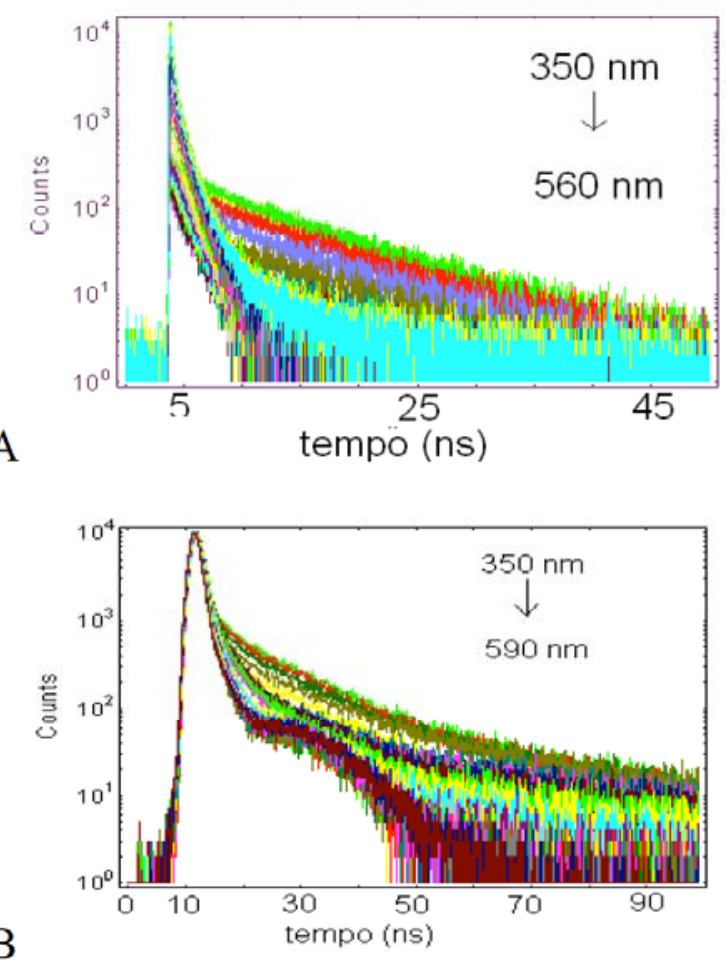

Time-resolved emission spectra (TRES) were also recorded for the blend in both temperatures, at room temperature and $77 \mathrm{~K}$. Spectra are shown in Figure 6. They were recorded at the range of 340 to $590 \mathrm{~nm}$, in a $10 \mathrm{~nm}$ increment, with excitation at $300 \mathrm{~nm}$. From these data, it is noteworthy that the fluorescence lifetime decreases as monitoring wavelengths are longer. Also, the shorter component of the lifetime decay curve becomes more important as the monitoring wavelength is longer. At room temperature, time-resolved spectra obtained from these decay curves show a very weak emission at 340 to $400 \mathrm{~nm}$, the carbazolyl-emitting region, and a strong emission at 500 to $700 \mathrm{~nm}$, corresponding to MEH-PPV emission which corroborates with the observed by the steadystate spectroscopic study. However, these data do not show the excimer emission occurring at $400-450 \mathrm{~nm}$. ON the other hand, when these spectra are obtained at $77 \mathrm{~K}$, a much intense emission in the carbazolyl region, similar in intensity to the emission from de MEH-PPV portion is detected. These observations reveal that at low temperatures, emission from the carbazolylcontaining matrix is more important than at room temperature and this may be due to the non-radiative energy transfer rate decrease from the carbazolyl units to the MEH-PPV units in the blend when system is frozen. This also maybe due to the more efficient carbazole triplet excited state population at lower
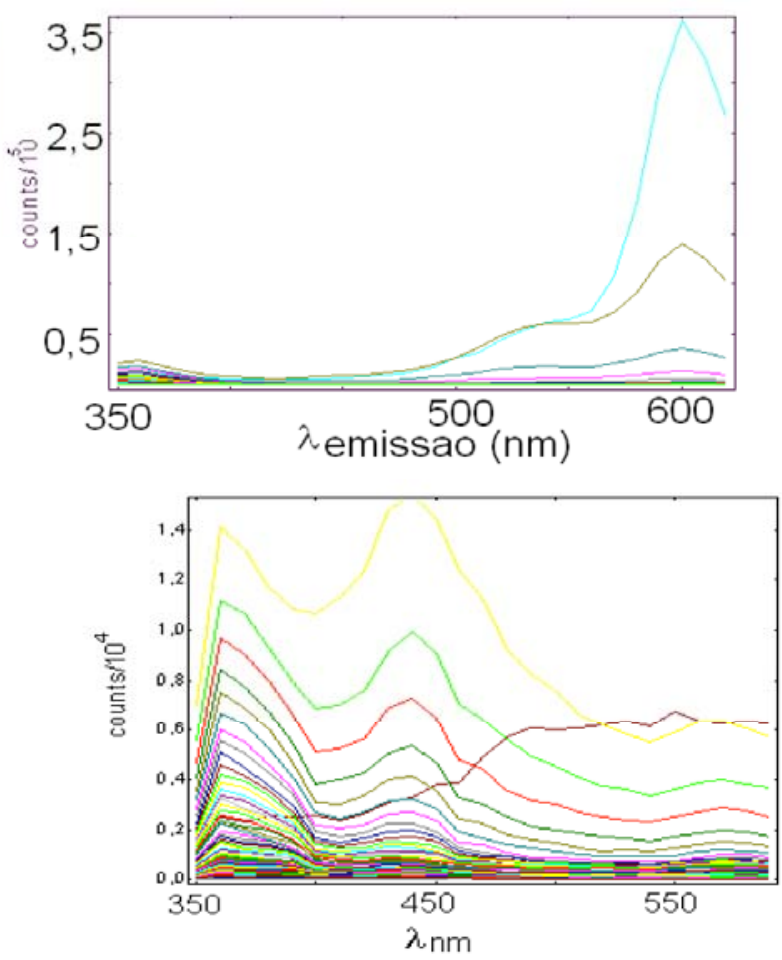

Figure 6: PMMA-Cz/ MEH-PPV fluorescence decay curves and time-resolved emission spectra $(\lambda$ exc $=300 \mathrm{~nm})$ recorded at $\mathbf{A})$ at room temperature and $\mathbf{B}$ ) at $77 \mathrm{~K}$. 
temperatures, which results in the phosphorescence emission that is observed. In this case, the most prominent energy transfer mechanism that occurs in this temperature range is the radiative energy transfer, not from isolated carbazolyl that emits at $350 \mathrm{~nm}$ region, but from light emitted consisting of two distinct sources: the excimer species that present fluorescence at $440 \mathrm{~nm}$ and from carbazolyl phosphorescence also at $440 \mathrm{~nm}$, both in the region of light-absorption of MEH-PPV.

\section{CONCLUSIONS}

Steady-state fluorescence spectroscopy was employed to describe the photophysical behavior of the PMMA-Cz/ MEH-PPV $(0.1 \% \mathrm{~m} / \mathrm{m})$ and the relaxation processes resulted of temperature effects. It was found that the relaxation processes are the same of the isolated components and that they are detectable when recording the fluorescence emission of the blend at the emission region of carbazolyl units or MEH-PPV units, indistinctly. Nevertheless, some probable minor processes could be discerned, which leaded to the careful miscibility investigation by using time-resolved fluorescence spectroscopy combined to fluorescence optical microscopy and scanning electronic microscopy. Shorter fluorescence lifetimes at the carbazolyl's emission region than those obtained for the same material when isolated, at room temperature and at $77 \mathrm{~K}$, indicate the occurrence of the energy transfer by a resonant process between excited states of carbazolyl, the donor, and those of the acceptor MEH-PPV, by Forster mechanism. SEM images showed the uniformity of the blend and FOM images revealed a degree of miscibility that is more important at lower temperatures, which, combined to the information of loss of intensity at the carbazolyl emission region and the shorter lifetimes at higher temperature, proved that energy transfer in this system is a result of a radiative and non-radiative mechanism, the latter prevailing at room temperature and being less important at $77 \mathrm{~K}$. Yet, at $77 \mathrm{~K}$, prevailing energy transfer mechanism is the trivial one, maybe enforced by the overlap of the phosphorescence emission of carbazolyl units and the absorption band of MEH-PPV portion.

\section{REFERENCES}

[1] Liu LJ, van Bavel S, Wen SP, Yang X, Loos J. Morphology and performance of poly(2-methoxy-5-(20-ethyl-hexyloxy)-pphenylenevinylene)(MEH-PPV):(6,6)-phenyl-C61-butyric acid methyl ester (PCBM) based polymer solar cells. Chinese $\mathrm{J}$ Chem 2013; 31(6): 731-5.

http://dx.doi.org/10.1002/cjoc.201300135
[2] Wu CS, Liu CT, Chen Y. Multifunctional copolyfluorene containing pendant benzimidazolyl groups: applications in chemical sensors and electroluminescent devices. Polym Chem 2012; 3(12): 3308-9.

http://dx.doi.org/10.1039/c2py20506h

[3] Parashchuk OD, Grigorian S, Levin EE, Bruevich VV, Bukunov K, Golovnin IV, Dittrich T, Dembo KA, Volkov VV, Paraschuk DY. Acceptor-Enhanced Local Order in Conjugated Polymer Films. J Phys Chem Lett 2013; 4(8): 1298-5.

http://dx.doi.org/10.1021/jz400333k

[4] Yang X, Wang RZ, Wang YC, Sheng CX, Li H, Hong W, Tang $\mathrm{WH}$, Tian CS, Chen Q. Long lived photoexcitation dynamics in pi-conjugated polymer and fullerene blended films. Org Electron 2013; 14(8): 2058-6. http://dx.doi.org/10.1016/j.orgel.2013.04.043

[5] Sosorev AY, Parashchuk, OD, Zapunidi SA, Kashtanov GS, Paraschuk DY. Intrachain Aggregation of Charge-Transfer Complexes in Conjugated Polymer:Acceptor Blends from Photoluminescence Quenching; J Phys Chem C 2013; 117(14): 6972-6.

http://dx.doi.org/10.1021/jp4000158

[6] Balderas U, Falcony C, Moggio I, Arias E, Mondragón M, A photoluminescence study of electrospun fibers of conjugated poly[2-methoxy-5-(20-ethylhexyloxy)-1,4-phenylenevinylene] blended with poly(9-vinylcarbazole). Polym 2013; 54: 2062 4. http://dx.doi.org/10.1016/i.polymer.2013.02.015

[7] Nicholson PG, Castro FA. Organic photovoltaics: principles and techniques for nanometre scale characterization. Nanotechnology 2010; 21(49): 492001.

http://dx.doi.org/10.1088/0957-4484/21/49/492001

[8] Peumans P, Yakimov A, Forrest SR, Small molecular weight organic thin-film photodetectors and solar cells. J Appl Phys 2003; 93(7): 3693-30 http://dx.doi.org/10.1063/1.1534621

[9] Martins TD, Weiss RG, Atvars TDZ. Synthesis and photophysical properties of a poly(methyl methacrylate) polymer with carbazolyl side groups. J Braz Chem Soc 2008; 19: $1450-14$ http://dx.doi.org/10.1590/S0103-50532008000800003

[10] Atvars TDZ, Talhavini M. Modifications in a spectrofluorometer for usage dedicated to polymer relaxation analysis. Quim. Nova 1991; 18: 298-3.

[11] Hoegl H. Photoelectric Effect in Polymers and Their Senstization by dopants. J Phys Chem 1965; 69: 755-11. http://dx.doi.org/10.1021/j100887a008

[12] Klopffer W. Luminescence of poly(n-vinyl-carbazole) films at 77K .2. kinetic-model of exciton trapping and annihilation. Chem Phys 1981; 57(1-2): 75-13. http://dx.doi.org/10.1016/0301-0104(81)80022-5

[13] $\mathrm{Ng} \mathrm{D}$, Guillet JE. Interpretation of the excimer kinetics of poly(nvinylcarbazo le)and 1,3-dicarbazolylpropane in dilute solutions. Macromolecules 1981; 14(2): 405-5.

\section{http://dx.doi.org/10.1021/ma50003a033}

[14] Somersall AC, Guillet EJ. Triplet-triplet annihilation and excimer fluorescence in poly(naphthyl methacrylate) Macromol 1973; 6(2): 218-5. http://dx.doi.org/10.1021/ma60032a013

[15] Soutar I, Swanson L, Davidson K, Yin J. Photophysics of carbazole-containing systems. 2. fluorescence behaviour of poly (n-vinyl carbazole) and n-vinyl carbazole/ methyl acrylate copolymer films, High Perform Polym 1997; 9: 353-15. http://dx.doi.org/10.1088/0954-0083/9/4/001

[16] Sakai H, Itaya A, Masuhara H, Sasaki K, Kawata S. Fluorescence dynamics of poly(n-vinylcarbazole) in fluid solution. multivariate analysis of time-resolved fluorescence spectra. Chem Phys Lett 1993; 208(3-4): 283-7.

http://dx.doi.org/10.1016/0009-2614(93)89076-T 
[17] Skilton PF, Ghiggino KP. Temperature-dependence of excimer formation in n-vinyl carbazole polymers, polymer photochem. Polym Photochem 1984; 5: 179-12. http://dx.doi.org/10.1016/0144-2880(84)90031-9

[18] Roberts AJ, Cureton CG, Philips D. Time-resolved emission studies of poly(n-vinyl carbazole) using pulsed laser excitation. Chem Phys Lett 1980; 72(3): 554-3. http://dx.doi.org/10.1016/0009-2614(80)80352-6

[19] Johnson GE. Emission properties of vinylcarbazole polymers. J Chem Phys 1975; 62(12): 4697-9.

http://dx.doi.org/10.1063/1.430418

[20] Roberts A J, Phillips D, Abdul-Rasoul FAM, Ladwith A. Temperature dependence of excimer formation and dissociation in poly( $\mathrm{N}$-vinylcarbazole). J Chem Soc Faraday Trans I 1981; 77: 2725-12. http://dx.doi.org/10.1039/f19817702725

[21] Gallego J, Mendicuti F, Mattice W. Energy migration in poly(n-vinyl carbazole) and its copolymers with methyl methacrylate: fluorescence polarization, quenching and molecular dynamics. J Polym Sci Part B: Polym Phys 2003; 41: 1615-12.

http://dx.doi.org/10.1002/polb.10508

[22] Buchberger EM, Mollay B, Weixelbaumer WD, Kauffmann HF. Excited state relaxation in bichromophoric rotors: timeresolved fluorescence of 1,3-di ( $n$-carbazolyl) propane: a three-state analysis. J Chem Phys 1988; 89(2): 635-18. http://dx.doi.org/10.1063/1.455239

[23] Itagaki H, Obukata N, Okamoto A, Horie K, Mita I. Kinetic studies on the formation of two intramolecular excimers in substituted dinaphthylpropanes. J Am Chem Soc 1982; 104: 4469-9. http://dx.doi.org/10.1021/ja00380a024

[24] Tagawa S, Washio M, Tabata Y. Picosecond time-resolved fluorescence studies of poly(n-vinylcarbazole) using a pulseradiolysistechnique. Chem Phys Lett 1979; 68(2-3): 276-6. http://dx.doi.org/10.1016/0009-2614(79)87200-0

[25] Giro G, Orlandi G. Temperature-dependence of the fluorescence-spectra of poly-n-vinylcarbazole films from 300$\mathrm{K}$ to $550-\mathrm{K}$ - evidence for monomer emission. Chem Phys 1992; 160: 145-6.

http://dx.doi.org/10.1016/0301-0104(92)87097-S

[26] Solaro R, Galli G, Masi F, Ledwith A, Chellini E. Synthesis, stereochemistry and fluorescence properties of polystyrenes having carbazole substituents. Eur Polym J 1983; 19(5): 4336. http://dx.doi.org/10.1016/0014-3057(83)90119-2

[27] Bonesi SM, Erra-Balselis R. Electronic spectroscopy of carbazole andnandc- substituted carbazoles in homogeneous media and in solid matrix. J Lumin 2001; 93: 51-24. http://dx.doi.org/10.1016/S0022-2313(01)00173-9

[28] Bree A, Zwarich R. Vibrational assignment of carbazole from infrared, raman, and fluorescence spectra. J Chem Phys 1968; 49(8): 3344-12.

http://dx.doi.org/10.1063/1.1670606

[29] Belletète $M$, Bouchard J, Leclerc $M$, Durocher G. Photophysics and solvent-induced aggregation of 2,7carbazole-based conjugated polymers. Macromolecules 2005; 38: 880-7.

http://dx.doi.org/10.1021/ma048202t

[30] Cossiello RFC, Kowalski E, Rodrigues PC, Akcelrud L, Bloise AC, DeAzevedo ER, Bonagamba TJ, Atvars TDZ. Photoluminescence and relaxation processes in MEH-PPV. Macromolecules 2005; 38(3): 925-8. http://dx.doi.org/10.1021/ma048340i

[31] Cossiello RF, Susman MD, Aramendia PF, Atvars TDZ. Study of solvent-conjugated polymer interactions by polarized spectroscopy: MEH-PPV and Poly(9,9dioctylfluorene-2,7-diyl). J Lumin 2010; 130: 415-9.

http://dx.doi.org/10.1016/j.jumin.2009.10.006
Becker-Guedes F, deAzevedo ER, Bonagamba TJ, SchmidtRohr K. Solid-state exchange nmr characterization of segmental dynamics in glassy poly(alkyl methacrylate)s Appl Mag Res 2004; 27 (3-4): 383-8.

[33] Christoff M, Atvars TDZ. Phosphorescent probes in studies of secondary relaxation of amorphous polystyrene and poly(nalkyl methacrylate)s. Macromolecules 1999; 32: 6093-9. http://dx.doi.org/10.1021/ma990008x

[34] De Deus JF, Souza GP, Corradini WA, Atvars TDZ, Akcelrud L. Photo and electroluminescence studies of poly(methyl methacrylate-co-9-anthryl methyl methacrylate) Macromolecules 2004; 37: 6938-6. http://dx.doi.org/10.1021/ma049941c

[35] Hejboer J, Baas MA, Van de Graaf B, Hoefnagel MA. A molecular mechanics study on rotational motions of side groups in poly(methyl methacrylate)Polymer, 1987, 28: 5095. http://dx.doi.org/10.1016/0032-3861(87)90207-2

[36] Williams J, Eisenberg A. Methyl-group tunneling and viscoelastic relaxation in poly(methyl methacrylate), Macromolecules 1978; 11: 700-8.

http://dx.doi.org/10.1021/ma60064a017

[37] Tanabe Y, Hirose J, Okano K, Wada Y. Methyl Group Relaxations in the Glassy Phase of Polymers. Polym J 1970; 1: 107-9. http://dx.doi.org/10.1295/polymj.1.107

[38] Baas JMA, Graaf VB, J. Heijboer J. A molecular mechanics investigation into the mechanism of the low temperature relaxations of three poly(alkyl methacrylates). Polymer 1991; 32: 2141-5.

http://dx.doi.org/10.1016/0032-3861(91)90037-J

[39] Amparo G, Soria V, Figueruelo JB, Calleja RD. dielectric and dynamic-mechanical relaxations in methyl methacrylate and methyl isopropenyl ketone copolymers. Polymer 1988; 29: 981-6.

http://dx.doi.org/10.1016/0032-3861(88)90004-3

[40] Deutsch H, Hoff EA, Reddish W. Relation between the structure of polymers and their dynamic mechanical and electrical properties. part I. some alpha-substituted acrylic ester polymers. J Polym Sci 1954; 13: 565-18.

http://dx.doi.org/10.1002/pol.1954.120137205

[41] Hyde PD, Evert TE, Cicerone MT, Ediger MD, Rotational motion of molecular probes in ortho-terphenyl and cispolyisoprene J Non-Cryst Solids 1991; 42: 131-17.

[42] Ehlich D, Sillescu $H$. Tracer diffusion at the glass transition. Macromolecules 1990; 23: 1600-11. http://dx.doi.org/10.1021/ma00208a008

[43] lyengar N, Harrison B, Duran RS, Schanze KS, Reynolds JR. Morphology evolution in nanoscale light-emitting domains in mehppv/pmma blends. Marcomolecules 2003; 36: 8978-8. http://dx.doi.org/10.1021/ma034908w

[44] Davidson K, Soutar I, Swanson L, Yin J. Photophysics of carbazole-containing systems. 1. Dilute solution behavior of poly (n-vinyl carbazole) and n-vinylcarbazole/ methyl acrylate copolymers. J Polym Phys 1997; 35: 963-15.

http://dx.doi.org/10.1002/(SICl)1099-

0488(19970430)35:6<963::AID-POLB11>3.0.CO;2-D

[45] Itaya A, Okamoto K, Kusabayashi S. Emission spectra of the vinyl polymers with pendant carbazolyl groups. Bull Chem Soc Jpn 1976; 49: 2082-7.

http://dx.doi.org/10.1246/bcsj.49.2082

[46] Förster T. 10th Spiers Memorial Lecture, Transfer Mechanism Of Electronic Excitation. Discuss Faraday Soc 1959; 27: 7-11.

http://dx.doi.org/10.1039/df9592700007

[47] Campbell IH, Smith DL. Consistent time of flight mobility measurements and polymer light-emitting diode currentvoltage characteristics. Appl Phys Lett 1999; 74: 2809-3.

http://dx.doi.org/10.1063/1.124021 
[48] Cariou JM, Dugas J, Martins L, Michel P. Refractive-index variations with temperature of PMMA and polycarbonate. Appl Opt 1986; 25(3): 334-3. http://dx.doi.org/10.1364/AO.25.000334
[49] Singh S. Refractive index measurement and its applications. Phys Script 2002; 65: 167-4.

http://dx.doi.org/10.1238/Physica.Regular.065a00167

Received on 01-10-2013

Published on 25-12-2013

DOI: http://dx.doi.org/10.12974/2311-8717.2013.01.01.8

(C) 2013 Martins and Atvars; Licensee Savvy Science Publisher.

This is an open access article licensed under the terms of the Creative Commons Attribution Non-Commercial License (http://creativecommons.org/licenses/by-nc/3.0/) which permits unrestricted, non-commercial use, distribution and reproduction in any medium, provided the work is properly cited. 